\title{
Histological and Biochemical Comparisons between Right Atrium and Left Atrium in Patients with Mitral Valvular Atrial Fibrillation
}

\author{
Jae Hyung Park, PhD, Jihei Sara Lee, BSc, Young-Guk Ko, MD, Seung Hyun Lee, MD, Beom Seob Lee, BSc, \\ Seok-Min Kang, MD, Byung Cheol Chang, MD, and Hui-Nam Pak, MD \\ Division of Cardiology, Yonsei University Health System, Seoul, Korea
}

Background and Objectives: It has been known that the dominant driver of atrial fibrillation (AF) exists in the left atrium (LA) and the incidence of systemic thromboembolism is higher than that of pulmonary thromboembolism in patients with AF. Therefore, we hypothesized that histological and biochemical characteristics of the LA and the right atrium (RA) are different in patients with mitral valvular AF. Subjects and Methods: We analyzed the histology and messenger ribonucleic acid (mRNA) or protein expression associated with endothelial function and thrombogenesis in 33 human atrial appendage tissues (20 LA tissues, 13 RA tissues) taken from 25 patients $\{57.7 \pm 11.3$ years old, $44 \%$ males, $A F$ : sinus rhythm $(S R)=17: 8\}$ with mitral valve disease. We also performed whole mRNA quantification in 8 tissues (both LA and RA tissues from 4 patients) by using next generation sequencing (NGS).

Results: 1) The degree of fibrosis ( $p=0.001)$ and subendocardial smooth muscle thickness $(p=0.004)$ were significantly greater in the $L A$ than in the RA. 2) More advanced matrix fibrosis was found in the LA of patients with AF than in the LA of patients with $S R(p=0.046)$, but not in the RA. 3) There was no LA-RA difference in protein (Western blot) and mRNA \{quantitative real-time polymerase chain reaction (qRT-PCR)\} expressions of NF- $\mathrm{KB}, 3-\mathrm{NT}, \mathrm{CD} 31$, E-selectin, inducible NO synthase, stromal cell-derived factor-1 $\alpha$, Endothelin-1, platelet-derived growth factor, myeloperoxidase, or NCX, except for higher mRNA expression of HCN4 in the RA (qRT-PCR, $p=0.026$ ) and that of KCNN1 in the LA (NGS, $p=0.016)$.

Conclusion: More advanced matrix and subendocardial remodeling were noticed in the LA than in the RA in patients with mitral valvular AF. However, the expressions of tissue factors associated with thrombogenesis were not significantly different between the RA and the LA. (Korean Circ J 2014;44(4):233-242)

KEY WORDS: Atrial fibrillation; Mitral valve disease; Fibrosis; Subendocardium.

\section{Introduction}

Atrial fibrillation (AF) is one of the most common cardiac arrhythmias. Over time, it has been understood as a prothrombotic disease. ${ }^{122)}$ According to Virchow's triad, three conditions must be present for thrombosis to occur, and all of the three conditions are present in AF: loss of atrial contractility, endocardial damage, and abnormalities in hemostatic variables. ${ }^{3)}$ However, although both atria fibrillate in AF, growing evidence suggests that the majority of thrombi originate in the left atrium (LA). Several studies have investigated the characteristics that make the LA more prone to thrombosis than the right atrium (RA) during AF. For example, Cai et al. ${ }^{4)}$ have reported that the level of nitric oxide (NO), a vasodilator and an antithrombotic agent, was markedly decreased in porcine $L A$ and LA appendage (LAA) during AF compared to that in LA showing sinus rhythm (SR), RA, and aorta. In line with the results of this study,

Received: February 15, 2014 / Revision Received: March 29, 2014 / Accepted: June 9, 2014

Correspondence:

Hui-Nam Pak, MD, Division of Cardiology, Yonsei University Health System, 50-1 Yonsei-ro, Seodaemun-gu, Seoul 120-752, Korea

Tel: 82-2-2228-8459,Fax: 82-2-2227-7732,E-mail: hnpak@yuhs.ac

Young-Guk Ko, MD, Division of Cardiology, Yonsei University Health System, 50-1 Yonsei-ro, Seodaemun-gu, Seoul 120-752, Korea

Tel: 82-2-2228-8460, Fax: 82-2-393-2041, E-mail: ygko@yuhs.ac

- The authors have no financial conflicts of interest.

This is an Open Access article distributed under the terms of the Creative Commons Attribution Non-Commercial License (http://creativecommons.org/licenses/ by-nc/3.0) which permits unrestricted non-commercial use, distribution, and reproduction in any medium, provided the original work is properly cited. 
Dudley et al. ${ }^{5)}$ found that the superoxide $\left(\mathrm{O}_{2}^{-}\right)$level was increased in the $L A$, suggesting greater NO degradation in the LA than in the RA during AF. Downstream of NO, several factors are known to be involved in inflammation, thrombosis, as well as in remodeling. Stromal cell-derived factor-1 (SDF-1) and endothelin-1 (ET-1) ${ }^{7)}$ are promoters of platelet activity ${ }_{i}^{8-10)}$ E-selectin and CD31 (PECAM) enable platelet aggregation and activation; ${ }^{1112)}$ and platelet-derived growth factor (PDGF) mediates fibrosis. ${ }^{13)}$ All of these molecules are, at least in part, regulated by N0.6)14) In addition, myeloperoxidase (MPO) was also recently found to mediate structural as well as electrical remodeling of atria during $A F^{15)}$ While a number of studies have attempted to compare between the LA and the RA, differences between the two chambers in terms of vessel wall characteristics still remain elusive. Therefore, in this study, we aimed to compare the degree of fibrosis and expression of $\mathrm{NO}$-mediated platelet activating proteins (pNF- $\kappa \beta, E T-1, S D F-1)$, inducible NO synthase (iNOS), proinflammatory proteins (E-selectin, CD31), and profibrotic proteins (MPO, PDGF) between the LA and the RA in an attempt to characterize the endocardial changes specific to the $L A$ during $A F$, and to elucidate the possible mechanism for thrombosis in fibrillating LA (Fig. 1).

\section{Subjects and Methods}

\section{Patient selection}

The study protocol was approved by the Institutional Review Board of Severance Cardiovascular Hospital, Yonsei University Health System. All of the patients provided written informed consent. The study enrolled 25 patients (mean age $57.7 \pm 11.3$ years, 44\% males), who underwent mitral valve surgery and biopsy of the LA or the RA appendage (Table 1). Among these patients, 23 had AF and 10 were in SR. Comprehensive transthoracic echocardiography was

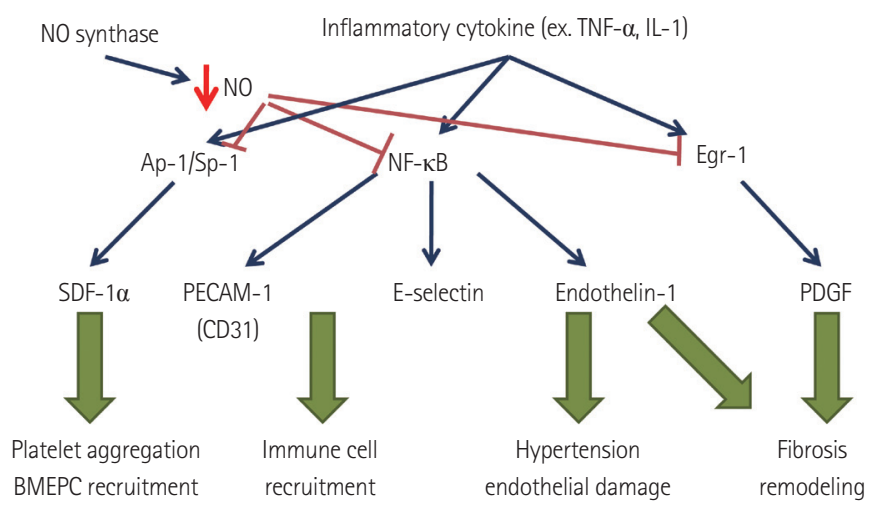

Fig. 1. Diagram of the mechanisms of multiple tissue factors associated with thrombosis. NO: nitric oxide, TNF: tumor necrosis factor, IL: interleukin, Ap-1/Sp-1: Ap-1/Sp-1 transcription factors, Egr-1: early growth response protein-1, SDF: stromal cell-derived factor, PECAM: platelet endothelial cell adhesion molecule, PDGF: platelet-derived growth factor, BMEPC: bone marrow-derived endothelial progenitor cells. performed using commercially available devices (Sonos 5500; Philips Medical System, Andover, MA, USA, or Vivid 7; GE Vingmed UItrasound, Horten, Norway). Standard M-mode, two-dimensional and Doppler images were acquired in the parasternal and apical views. Left atrial (LA) anterior-posterior diameter was measured, and left ventricular (LV) end-diastolic dimension (LVEDD) and LV end-systolic dimension (LVESD) were calculated from two-dimensional images using the modified Quinones method. Full wall thickness specimens were excised from the LA or the RA appendages. All of the myocardial specimens were fixed in 70\% alcohol and 10\% formalin solution immediately after excision.

\section{Immunohistochemical studies}

Multiple $5 \mu \mathrm{m}$ thick serial sections were used. Masson's trichrome (MT) stain was used to determine the presence and degree of fibrosis. Immunohistochemical staining was performed using an avidinbiotin peroxidase system (Dako, Carpinteria, CA, USA). Paraffin-embedded tissue sections were deparaffinized and then washed with phosphate-buffered saline (PBS). Hydrogen peroxidase block (Dako, Carpinteria, CA, USA) was placed on the sections for 10 minutes, and the slides were washed in PBS. Protein block (Dako, Carpinteria, CA, USA) was placed on the sections for 30 minutes. Primary antibodies were then incubated for 90 minutes at room temperature (approx. $25^{\circ} \mathrm{C}$ ). Antibodies for $\alpha$-SMA (1:500 dilution, rabbit anti-human polyclonal; Abcam) were used to stain the smooth muscle. After incubation, the slides were washed in PBS, and the appropriate secondary antibody (Dako, Carpinteria, CA, USA) was placed on the sections for 30 minutes. The sections were washed again in PBS, and the appropriate chromagen was added to each specimen. Smooth mucle cells and gap junctions were stained brown with 3,3'-diaminobenzidine (DAB). Cell nuclei were marked by placing the specimens in hematoxylin (Merck) for 1 minute. The specimens were then dehydrated in alcohol, mounted, and examined under light microscopy. The specimens stained with DAB were examined by light microscopy and virtual microscopy. Histopathological slides were analyzed by use of a computer-assisted morphometric analysis system (Image-Pro Plus 6.0; Media Cybernetics, Silver Spring, MD, USA).

The selection and analyses of virtual microscopic images were performed by a single technician, who was blinded to the experimental information of histological slides obtained with a consistent method. To quantify the fibrotic area, we selected the virtual microscopic fields with the fibrotic area on MT staining, and took images at a 160 objective in the quadrants of each slide. In each of the four fields, the smooth muscle area was identified as fibrillar structures positive for $\alpha$-SMA immunohistostaining, between myocardial cells, which were larger than $10 \mu \mathrm{m}$ and stained brown (RGB values: Red 22-125, Green 4-77, and Blue 4-55). In the endocardium, 
Table 1. Clinical characteristics

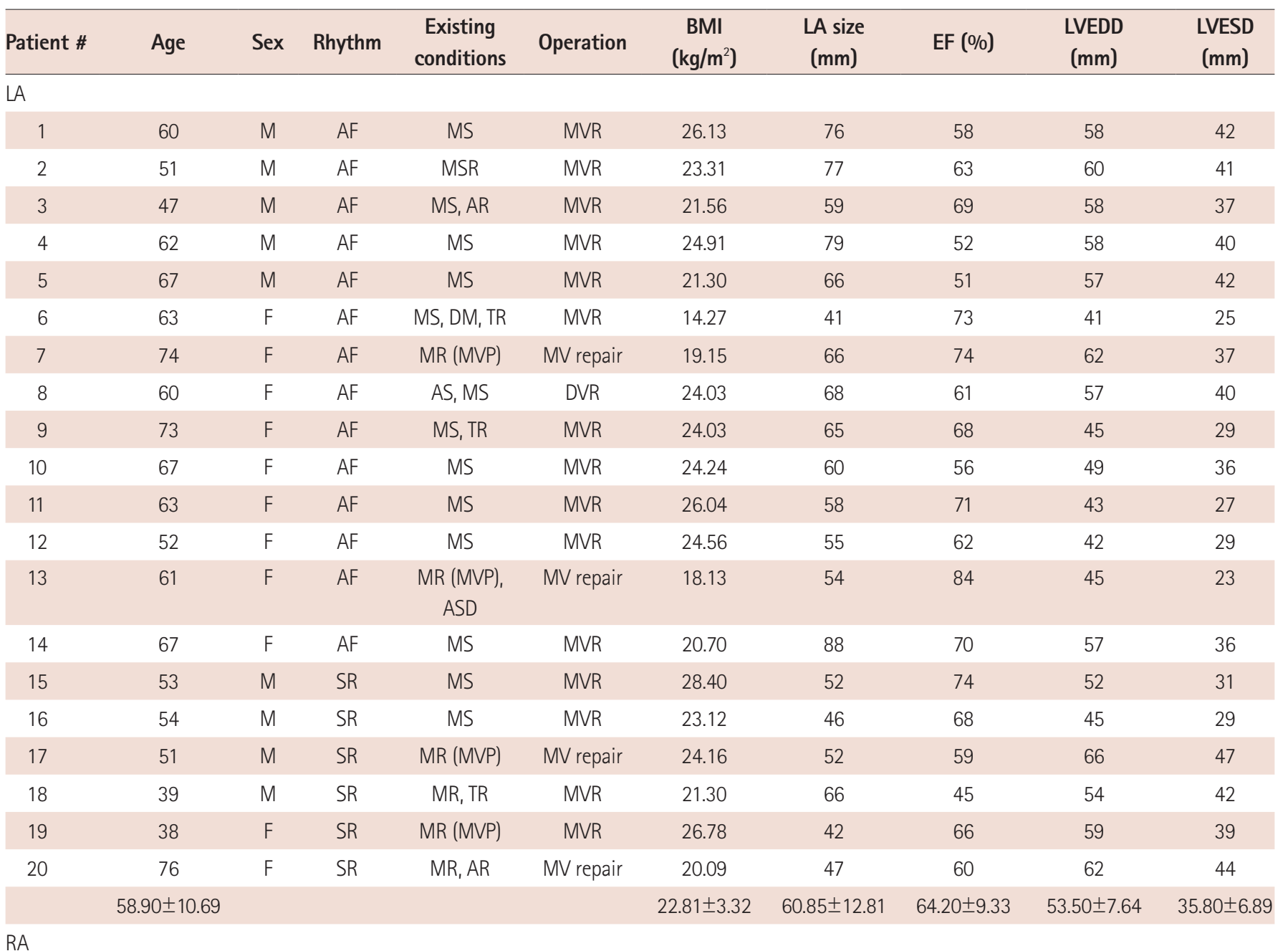

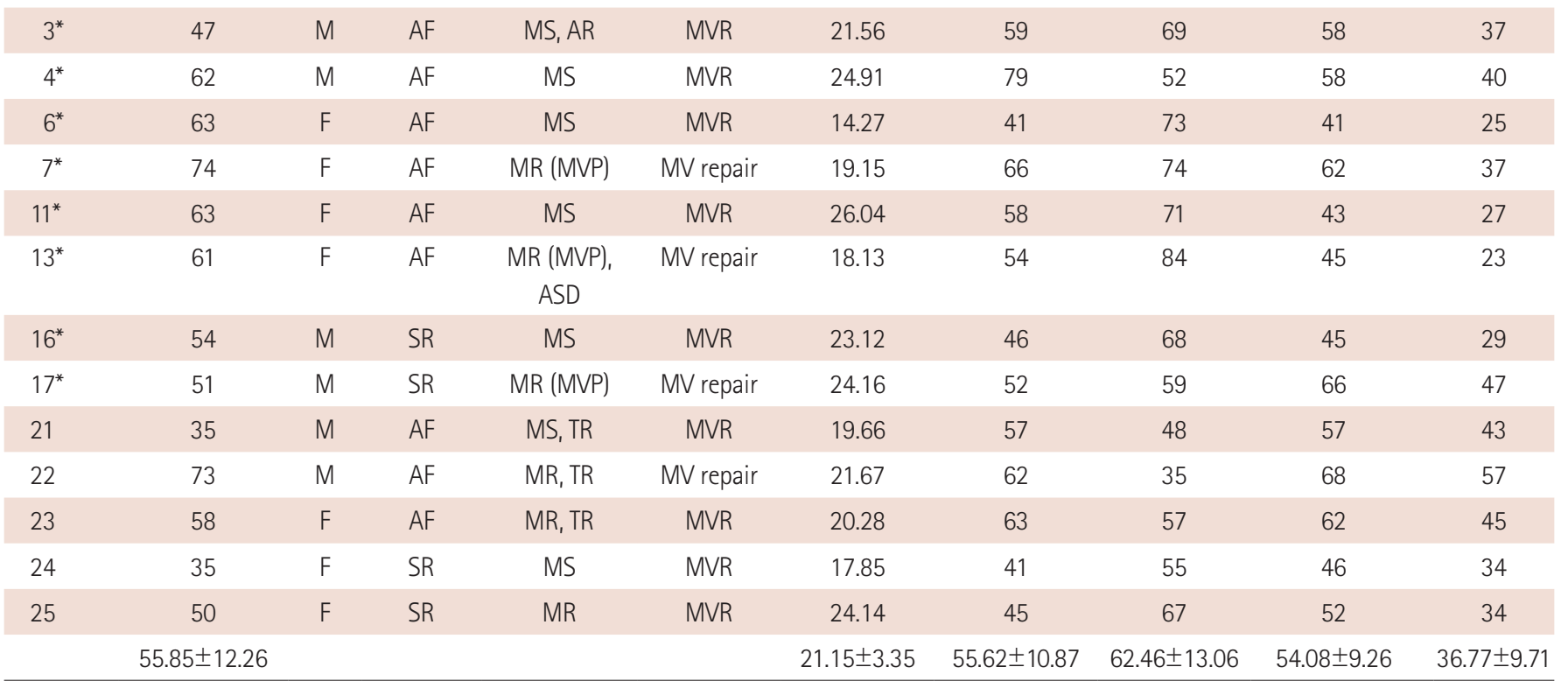

*Overlapping patients in both LA and RA groups. LA: left atrium, RA: right atrium, M: male, F: female, AF: atrial fibrillation, SR: sinus rhythm, MS: mitral stenosis, MR: mitral regurgitation, MVP: mitral valve repair, AR: aortic regurgitation, TR: tricuspid regurgitation, ASD: atrial septal defect, MVR: mitral valve replacement, MV repair: mitral valve repair, DVR: double valve replacement, EF: ejection fraction, LVEDD: left ventricular end-diastolic dimension, LVESD: left ventricular end-systolic dimension, BMI: body mass index 
we measured the subendocardial thickness and smooth muscle thickness maintaining distance of $200 \mu \mathrm{m}$. The percent immunostained areas and distance of the fibrotic area, and $\alpha$-SMA-positive smooth muscle cells were quantified using Image Pro software 6.0 (Media Cybernetics Inc., Silver Spring, MD, USA). The virtual histology was then analyzed as described before. ${ }^{16)}$ The percent area of fibrosis was quantified with MT staining utilizing the Image Pro software.

\section{Real-time quantitative polymerase chain reaction}

The method used to quantitate cDNA was the quantitative realtime polymerase chain reaction (PCR) method. The levels of cDNA were determined via fluorescence by using the thermal cycler during the PCR cycle. The real-time PCR reactions were performed using SYBR 1 Green Kit in a $20 \mu \mathrm{L}$ reaction volume containing 50 ng of cDNA. PCR reactions were carried out using a sequence detection system. Thermal cycling and SYBR Green fluorescence detection were performed using a real-time PCR thermal cycler (ABI PRISM 7500 Sequence Detection System, Applied Biosystems, Foster City, CA, USA). The primers were designed for HCN4 (Forward: GCAGC GCATCCACGACTAC, Reverse: TTAGCTCGCCCAGGATGCT), and NCX (Forward: TTCCAGAATGATGAAATTGTGAAGAT, Reverse: TCCTCAAG CACAAGGGAGAAAC) using the primer express software. The sodium calcium exchanger (NCX) forward primer ( $5^{\prime}$ to $3^{\prime}$ ) was TTCCAGA ATGATGAAATTGTGAAGAT and the reverse primer ( $5^{\prime}$ to $3^{\prime}$ ) was TCC TCAAGCACAAGGGAGAAAC. Then, $20 \mu \mathrm{L}$ PCR reactions were prepared in 96 well plates with a computer-assisted robotic and liquid handling automated workstation. We chose HCN4 and NCX because they play important roles in arrhythmogenicity and embryological development of the RA and the LA. Thermal cycling conditions were as follows: $50^{\circ} \mathrm{C}$ for 2 minutes and $95^{\circ} \mathrm{C}$ for 10 minutes, followed

Table 2. Clinical and histological characteristics of the $L A$ and the RA

\begin{tabular}{|c|c|c|c|}
\hline & LA $(n=20)$ & $\mathrm{RA}(\mathrm{n}=13)$ & $p$ \\
\hline Age & $58.90 \pm 10.69$ & $55.85 \pm 12.26$ & 0.455 \\
\hline Sex (male, \%) & 45.00 & 42.86 & 0.950 \\
\hline $\mathrm{BMI}\left(\mathrm{kg} / \mathrm{m}^{2}\right)$ & $22.81 \pm 3.32$ & $21.15 \pm 3.35$ & 0.172 \\
\hline $\mathrm{BSA}\left(\mathrm{m}^{2}\right)$ & $1.64 \pm 0.22$ & $1.59 \pm 0.23$ & 0.529 \\
\hline \multicolumn{4}{|l|}{ Echocardiographic findings } \\
\hline LA size $(\mathrm{mm})$ & $60.85 \pm 12.81$ & $55.62 \pm 10.87$ & 0.234 \\
\hline LA volume $(\mathrm{mL})$ & $179.69 \pm 101.22$ & $139.75 \pm 67.97$ & 0.221 \\
\hline LA volume Index $\left(\mathrm{mL} / \mathrm{m}^{2}\right)$ & $130.70 \pm 73.20$ & $91.84 \pm 33.64$ & 0.132 \\
\hline $\operatorname{LVEF}(\%)$ & $64.20 \pm 9.33$ & $62.46 \pm 13.06$ & 0.658 \\
\hline $\mathrm{E} / \mathrm{Em}$ & $16.39 \pm 5.89$ & $18.46 \pm 5.17$ & 0.554 \\
\hline LVEDD (mm) & $53.50 \pm 7.64$ & $54.08 \pm 9.26$ & 0.847 \\
\hline LVESD (mm) & $35.80 \pm 6.89$ & $36.77 \pm 9.71$ & 0.739 \\
\hline \multicolumn{4}{|l|}{ Histologic analyses } \\
\hline Fibrosis (MT) (\% area) & $23.78 \pm 10.22$ & $11.33 \pm 6.37$ & 0.001 \\
\hline Subendocardial smooth muscle area (\%) & $4.39 \pm 5.40$ & $0.32 \pm 0.58$ & 0.011 \\
\hline Subendocardial smooth muscle thickness (mm) & $0.16 \pm 0.17$ & $0.01 \pm 0.01$ & 0.004 \\
\hline \multicolumn{4}{|l|}{ Western blot } \\
\hline$p N F-k \beta$ & $1.22 \pm 0.38$ & $1.02 \pm 0.31$ & 0.198 \\
\hline CD31 & $0.70 \pm 0.20$ & $0.78 \pm 0.23$ & 0.420 \\
\hline E-selectin & $0.97 \pm 0.42$ & $0.17 \pm 0.35$ & 0.145 \\
\hline iNOS & $0.59 \pm 0.39$ & $0.62 \pm 0.44$ & 0.876 \\
\hline SDF- $1 \alpha$ & $0.74 \pm 0.37$ & $0.61 \pm 0.22$ & 0.380 \\
\hline Endothelin-1 & $0.86 \pm 0.27$ & $0.71 \pm 0.24$ & 0.218 \\
\hline PDGF & $0.53 \pm 0.25$ & $0.45 \pm 0.06$ & 0.528 \\
\hline MPO & $0.74 \pm 0.28$ & $0.93 \pm 0.55$ & 0.508 \\
\hline \multicolumn{4}{|l|}{ Real-time PCR } \\
\hline $2^{-\triangle C t N C X}$ & $0.012 \pm 0.012$ & $0.007 \pm 0.005$ & 0.139 \\
\hline $2^{-\triangle \mathrm{Ct} H C N 4}$ & $0.011 \pm 0.010$ & $0.027 \pm 0.028$ & 0.026 \\
\hline
\end{tabular}

LA: left atrium, RA: right atrium, BMI: body mass index, BSA: body surface area, LVEF: left ventricular ejection fraction, LVEDD: left ventricular end-diastolic dimension, LVESD: left ventricular end-systolic dimension, MT: Masson's trichrome, iNOS: inducible NO synthase, SDF-1: stromal cell-derived factor-1, PDGF: platelet-derived growth factor, MPO: myeloperoxidase, PCR: polymerase chain reaction 
by 40 cycles at $95^{\circ} \mathrm{C}$ for 30 seconds, $60^{\circ} \mathrm{C}$ for 30 seconds and $72^{\circ} \mathrm{C}$ for 1 minute. A melting curve analysis was performed after the final PCR cycle to evaluate the presence of nonspecific PCR products and primer dimers. During the initial optimization run, four-fold serial dilution was employed to demonstrate the linear amplification range for each gene. The threshold cycle (Ct) represents the PCR cycle during which an increase in SYBR Green fluorescence above the baseline signal can be detected first. For each sample, the amount of messenger ribonucleic acid (mRNA) was quantified relative to $0.5 \mu \mathrm{g}$ of total RNA applied by real-time PCR. Relative quantitation was performed by normalizing $\mathrm{Ct}$ values of each sample gene to $\mathrm{Ct}$ values of the housekeeping gene ( $\Delta \mathrm{Ct})$. $\Delta \mathrm{Ct}$ corresponds to the difference between the $\mathrm{Ct}$ of the gene of interest and the $\mathrm{Ct}$ of the housekeeping gene. Also, we tried to perform RNA Sequencing on transformed human tissues.

\section{Next generation sequencing}

To compare the amount of proteins and mRNA, we performed whole mRNA quantification in 8 tissues (4 from the LA, 4 from the RA) by using the next generation sequencing (NGS) method. Sequencing library was prepared using the NEBNext RNA prep kit (NEB, MA, USA). Briefly, the poly-A containing mRNA molecules were purified using poly-T oligonucleotide-attached magnetic beads. The mRNA was fragmented into small pieces and copied into cDNA. Library preparation process including end-repairing and subsequent adapter ligation produced the final library with size ranging from 250-350 bp. Sequencing was performed using HiSeq 2500 (Illumina, CA, USA) with the rapid run 150 bp PE mode. ${ }^{17)}$

\section{Western blot analysis}

For protein analysis, previously excised human LA tissues stored in liquid nitrogen $\left(-80^{\circ} \mathrm{C}\right)$ were used. The tissue specimens were homogenized and lysed in a mixture of $50 \mathrm{mmol}$ Tris ( $\mathrm{pH} 7.4), 5$ mM EDTA (pH 8.0), and 1\% Triton X-100. The samples were centrifuged at $13000 \mathrm{rpm}$ for 1 hour at $4^{\circ} \mathrm{C}$ to separate the supernatant. Protein content was determined by the BCA assay at $55^{\circ} \mathrm{C}$. Extracts were separated by SDS-polyacrylamide gel electrophoresis and transferred to polyvinylidene difluoride membranes. After blocking in TBS-Tween 20 (TBS-T, 0.1\% tween 20) containing 5\% non-fat dry milk, the membrane was incubated overnight with primary antibodies; pNF-к $\beta$ (1:1000 dilution, mouse antihuman monoclonal; Cell Signaling), CD31 (1:1000 dilution, rabbit antihuman polyclonal; Abcam, Cambridge, MA, USA), E-selectin (1:1000 dilution, rabbit antihuman polyclonal; Abcam, Cambridge, MA, USA), iNOS (1:1000 dilution, Abcam, Cambridge, MA, USA), SDF-1 $\alpha$ (1:1000 dilution, rabbit antihuman polyclonal; Abcam, Cambridge, MA, USA), ET-1 (1:1000 dilution, mouse antihuman monoclonal; Thermo Scientific, Hudson, $\mathrm{NH}$, USA), PDGF (1:500 dilution, rabbit antihuman polyclonal; Pierce Biotechnology, Rockford, IL, USA), and MPO (1:1000 dilution, rabbit antihuman polyclonal; Abcam, Cambridge, MA, USA). The primary antibody incubation was followed by incubation with HRP-conjugated secondary antibodies (Dako, Carpinteria, CA, USA). The antigen-antibody bands were detected using the enhanced chemiluminescence reagent kit (Millipore Co, Bedford, MA, USA) and quantified by densitometry.

\section{Data analysis}

Data are expressed as mean \pm SD. We conducted several comparison

\section{A MT stain}
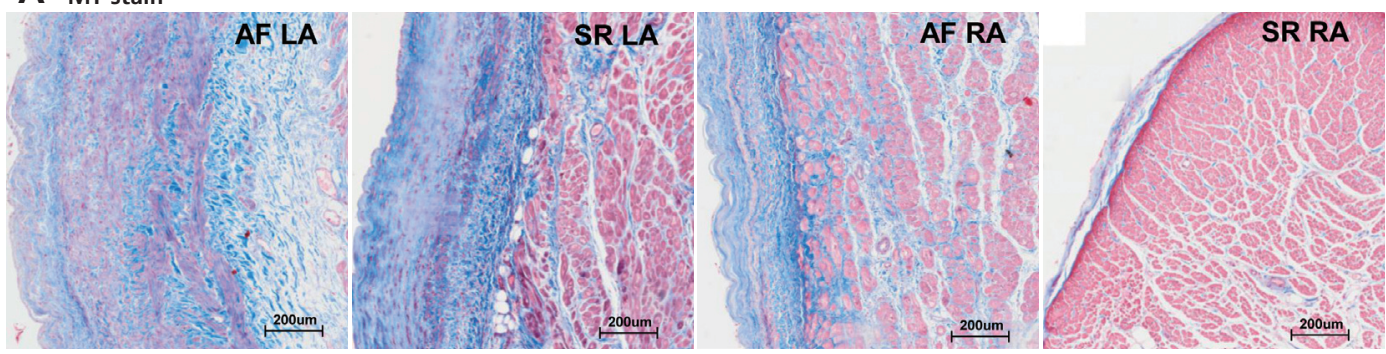

\section{B $\alpha$-SMA}
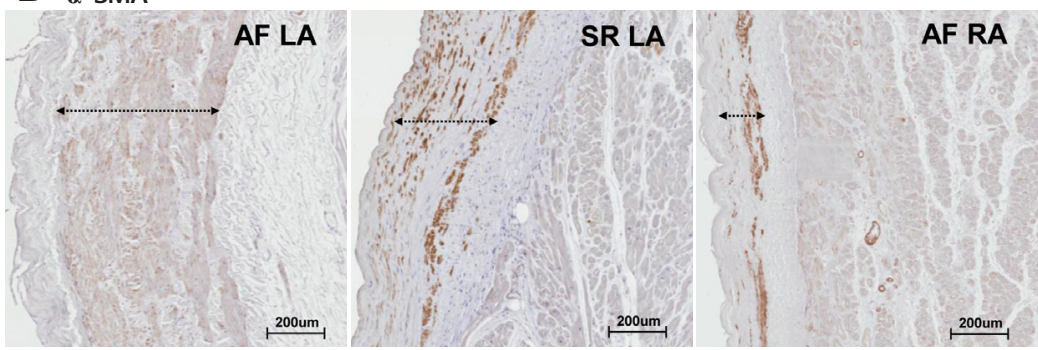

SR RA

Fig. 2. Masson's trichrome (MT) staining (A) and $\alpha$-smooth muscle actin (SMA) staining (B) of AF-LA, SR-LA, AF-RA, and SR-RA. AF: atrial fibrillation, LA: left atrium, RA: right atrium, $S R$ : sinus rhythm. 
analyses on thickness of the subendocardial smooth muscle layer, the fibrotic area, the density of the protein bands in the western blot analysis, and real-time PCR result in various cohorts. The statistical significance of these comparisons was assessed using the Student's t-test or the paired t-test. $p$ less than 0.05 were considered statistically significant.

\section{Results}

\section{Severe remodeling of the left atrium compared to the right atrium in patients with mitral valve disease}

The baseline characteristics of enrolled patients are summarized in Table 1. We divided the patients into a LA group $(n=20)$ and a
RA group ( $n=13)$ and compared their clinical and histological characteristics (Table 2). The data showed no significant difference in their clinical and echocardiographic characteristics. The percent areas of subendocardial fibrosis (MT stain, $p=0.001$ ) and subendocardial smooth muscle layer ( $\alpha$-SMA immunostaining, $p=0.011$ ) were greater in the LA than in the RA (Fig. 2). Subendocardial smooth muscle thickness was also higher in the LA than in the RA; thus demonstrating greater structural remodeling in the LA than in the RA $(p=0.004)$ (Table 2).

\section{Differential degree of left atrium remodeling in patients with atrial fibrillation and patients in sinus rhythm}

We divided the patients with mitral valve disease into patients

Table 3. Clinical and histological characteristics of AF patients' LA and RA and that of SR controls' LA and RA

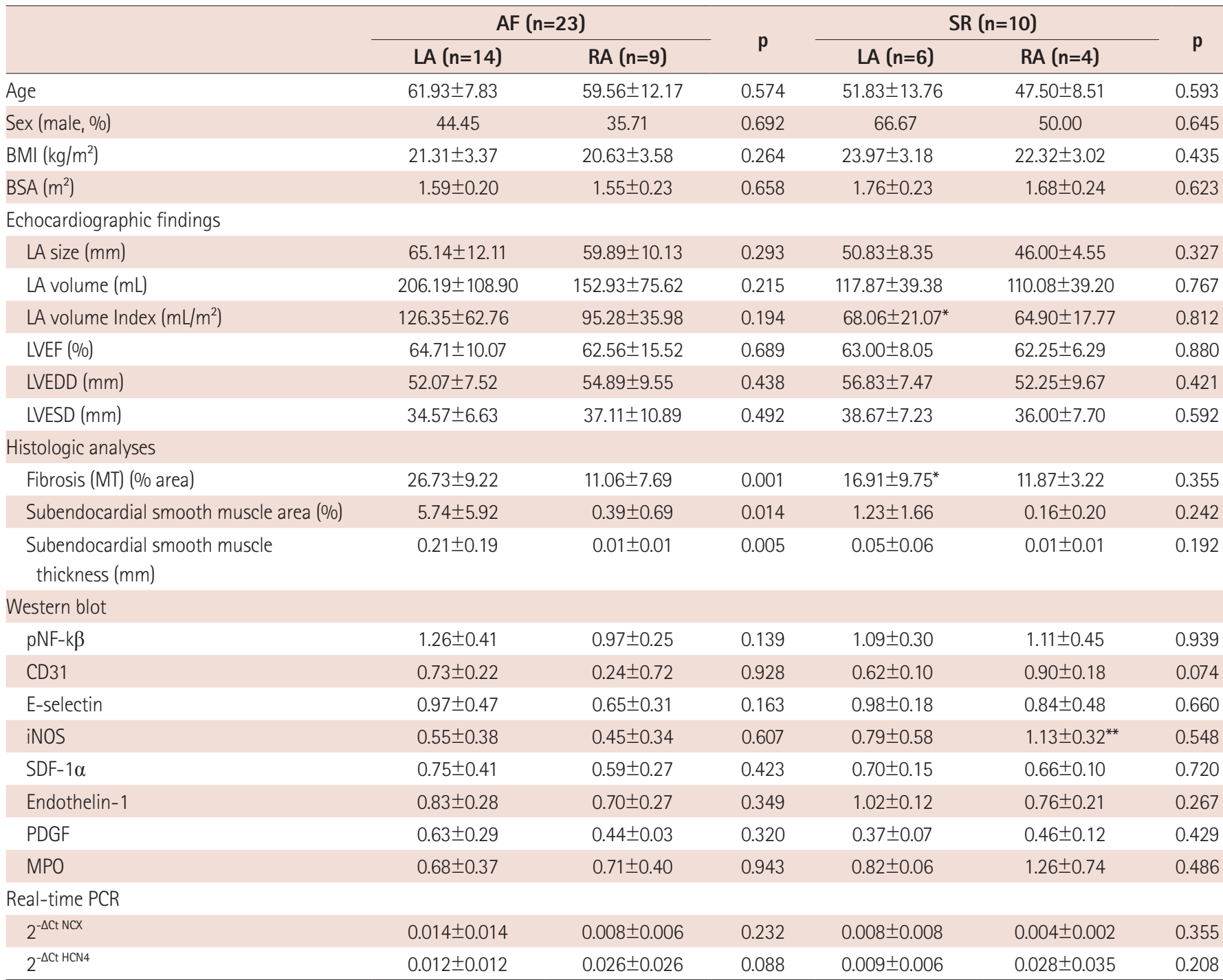

${ }^{*} p<0.05$ AF LA vs. SR LA, ${ }^{* *} p<0.05$ AF RA vs. SR RA. AF: atrial fibrillation, LA: left atrium, RA: right atrium, SR: sinus rhythm, BMI: body mass index, BSA: body surface area, LVEF: left ventricular ejection fraction, LVEDD: left ventricular end-diastolic dimension, LVESD: left ventricular end-systolic dimension, MT: Masson's trichrome, iNOS: inducible NO synthase, SDF-1: stromal cell-derived factor-1, PDGF: platelet-derived growth factor, MPO: myeloperoxidase, PCR: polymerase chain reaction 
with $A F$ and patients in $S R$, and then, subdivided the AF and SR groups into the LA and RA groups (Table 3 ). The clinical analysis revealed that the 4 groups showed no significant differences in sex, age, LA size, and LA volume. In terms of the degree of fibrosis, LA of the patients with AF showed more advanced interstitial fibrosis
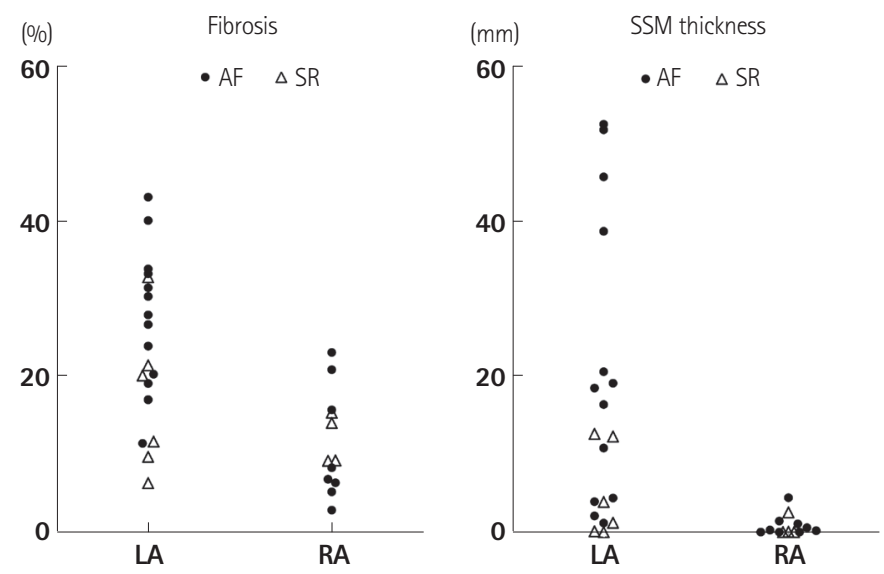

Fig. 3. Scatter plots of fibrosis area (\%) and thickness of the subendocardial smooth muscle layer $(\mathrm{mm})$ for the LA and RA. Most of the remodeling occurred in the fibrillating LA followed by LA showing sinus rhythm. AF: atrial fibrillation, LA: left atrium, RA: right atrium, SR: sinus rhythm.
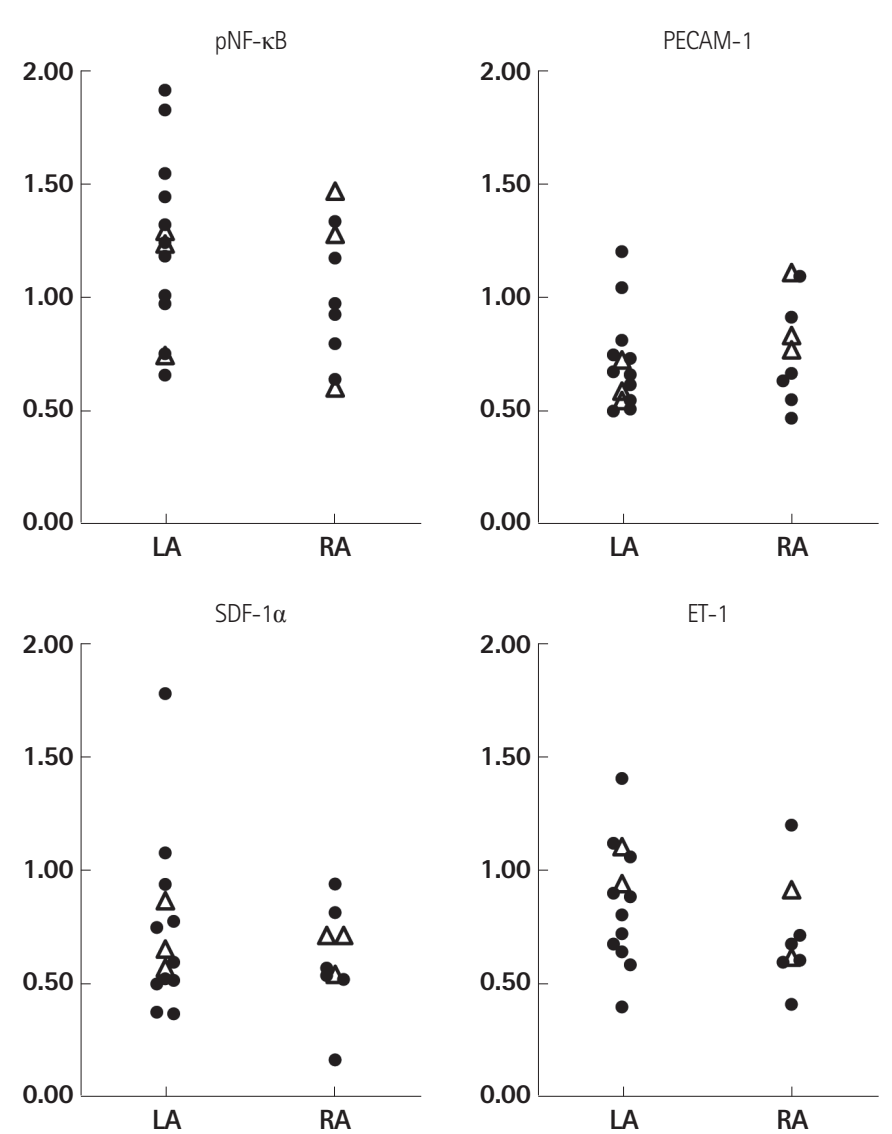

than LA of the patients in SR ( $p=0.046)$. But, there was no difference in the degree of fibrosis in the RA regardless of the rhythm status $(p=0.355)$. Among the patients with mitral valvular $A F$, the degree of fibrosis ( $p=0.001)$, percent area $(p=0.014)$ and thickness $(p=0.005)$ of the subendocardial smooth muscle layer were significantly greater in the LA than in the RA (Fig. 3).

\section{The messenger ribonucleic acid or protein expression level in the left atrium and right atrium tissues}

We have summarized the tissue characteristics based on the protein and mRNA expressions in Table 2. The protein expressions analyzed by western blot revealed that there were no significant differences in pNF- $\kappa B, C D 31$, E-selectin, iNOS, SDF-1 $\alpha, E T-1, P D G F$, and MPO expressions between the LA and the RA groups (Fig. 4). The tissue expression levels of thrombogenicity-related proteins were not significantly different among the 4 groups; AF vs. SR and $L A$ vs. RA, except for the lower tissue level of iNOS in the LA of patients in SR than in the RA of patients in SR ( $p=0.048$ ) (Table 3). In terms of mRNA expression, NGS for mRNA was conducted using 8 tissues taken from 4 patients ( $2 \mathrm{AF}$ and 2 patients remained in sinus rhythm), and all these were patient-matched $R A(n=4)$ and $L A$
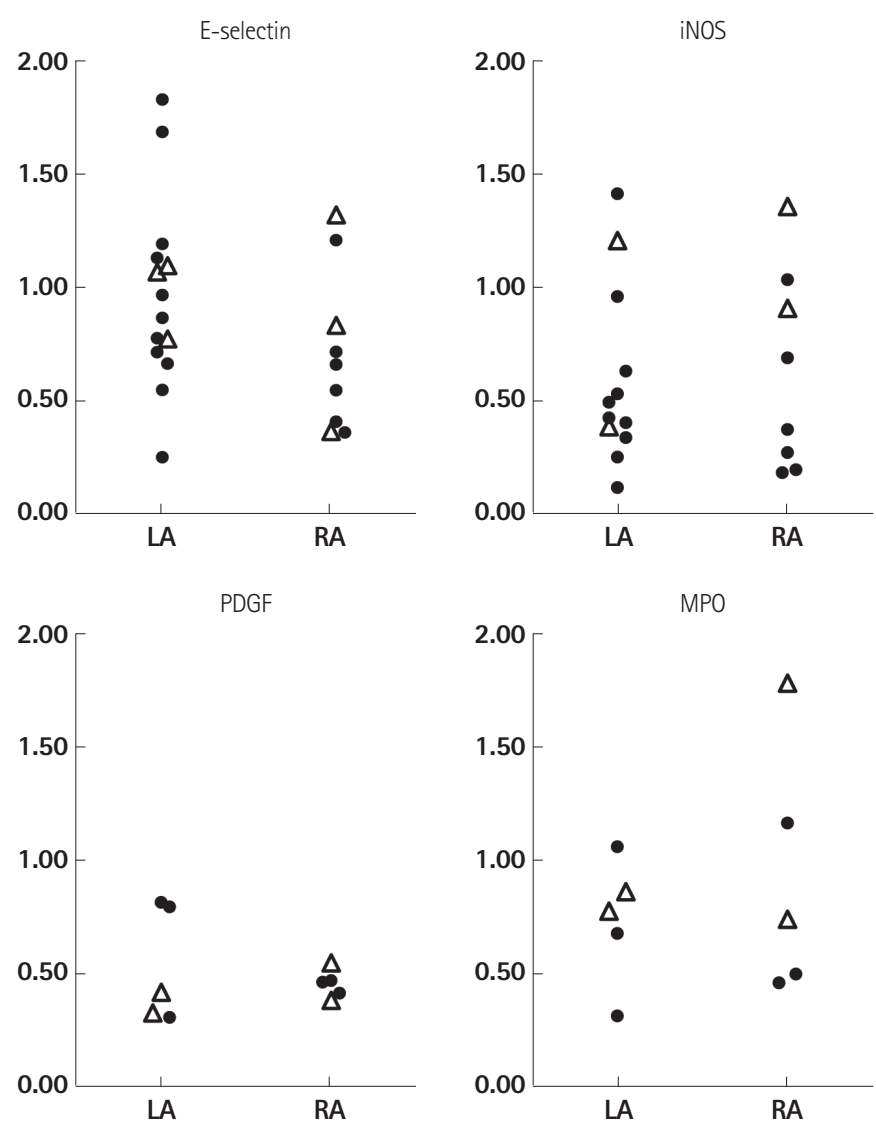

Fig. 4. Western blot analysis of pNF- $\kappa B, C D 31$, E-selectin, iNOS, SDF- $1 \alpha$, Endothelin-1, PDGF, and MPO showed that the protein expression levels were not significantly different between the LA and the RA. LA: left atrium, RA: right atrium, iNOS: inducible NO synthase, SDF-1: stromal cell-derived factor-1, PDGF: platelet-derived growth factor, MPO: myeloperoxidase. 
Table 4. Next generation sequencing of mRNAs associated with tissue thrombogenicity

\begin{tabular}{|c|c|c|c|c|c|c|}
\hline & LA $(n=4)$ & $\mathrm{RA}(n=4)$ & p & $\mathrm{AF}(n=4)$ & $\operatorname{SR}(n=4)$ & p \\
\hline Nuclear factor of kappa light polypeptide gene enhancer in B-cells 1 & $1.32 \pm 1.32$ & $1.49 \pm 0.73$ & 1.000 & $1.49 \pm 1.15$ & $1.40 \pm 0.85$ & 0.697 \\
\hline Platelet/endothelial cell adhesion molecule 1 & $10.87 \pm 3.55$ & $11.71 \pm 2.68$ & 0.269 & $8.51 \pm 5.91$ & $13.06 \pm 2.19$ & 0.707 \\
\hline Nitric oxide synthase 1 (neuronal) & $0.02 \pm 0.02$ & $0.03 \pm 0.01$ & 0.955 & $0.02 \pm 0.01$ & $0.03 \pm 0.01$ & 0.399 \\
\hline Stromal cell-derived factor 2 & $4.69 \pm 3.00$ & $4.44 \pm 2.89$ & 0.547 & $4.32 \pm 3.38$ & $4.68 \pm 2.73$ & 0.741 \\
\hline Platelet-derived growth factor alpha polypeptide & $4.93 \pm 3.22$ & $5.56 \pm 4.14$ & 0.639 & $4.20 \pm 3.95$ & $6.01 \pm 3.72$ & 0.946 \\
\hline Myeloperoxidase & $0.04 \pm 0.01$ & $0.04 \pm 0.02$ & 0.298 & $0.04 \pm 0.01$ & $0.04 \pm 0.02$ & 0.297 \\
\hline $\begin{array}{l}\text { Potassium intermediate/small conductance calcium-activated } \\
\text { channel, subfamily N, member } 1 \text { (KCNN1) }\end{array}$ & $0.21 \pm 0.10$ & $0.02 \pm 0.05$ & 0.016 & $0.11 \pm 0.16$ & $0.12 \pm 0.10$ & 0.919 \\
\hline
\end{tabular}

mRNA: messenger ribonucleic acid, AF: atrial fibrillation, LA: left atrium, RA: right atrium, SR: sinus rhythm

tissues $(n=4)$. It showed no significant difference in the mRNA levels of thrombogenicity-related proteins between the LA and the RA tissues or between patients with AF and patients in SR (Table 4). The mRNA expression of $\mathrm{HCN} 4$, a membrane ion channel protein, as measured by real time-PCR was significantly higher in RA tissues than in LA tissues ( $p=0.026$ ) (Table 2), but that of KCNN1 (calcium activated potassium channel subfamily) was higher in LA tissues than in RA tissues ( $p=0.016$ ) (Table 4). The mRNA expression of NCX was not significantly different between the LA and RA tissues $(p=0.139)$.

\section{Discussion}

In the current study, we report that fibrosis and subendocardial smooth muscle thickness were significantly greater in the LA than in the RA of patients with AF. Also, matrix fibrosis was more advanced in the LA of patients with AF than that in the LA of patients in SR. However, we did not observe any difference in the factors that contribute to vessel wall abnormality in thrombosis between the LA and the RA. To the best of our knowledge, this is the first study to systematically analyze the differences in endocardial characteristics between the LA and the RA in patients with AF and to exclude tissue factors as a cause of enhanced thrombogenesis in the fibrillating LA.

\section{Atrial fibrillation-related endocardial remodeling and dysfunction}

As the number of studies investigating the endocardium in the setting of AF has increased, AF is being increasingly recognized to exhibit endocardial dysfunction and damage. With the onset of AF, ensuing inflammation increases the expression of cell adhesion molecules such as E-selectin on the endocardial surface. ${ }^{18)}$ Moreover, anti-coagulant mechanism of the endocardium is attenuated when ET-1 is increased. ${ }^{7)}$ Ultimately, a pro-thrombotic environment is formed in the fibrillating atria. Furthermore, inflammatory molecules like C-reactive protein induce endocardial cells to increase the expression of PDGF. ${ }^{19)}$ In response, fibroblasts proliferate and alter the extracellular matrix. ${ }^{20)}$ Structural remodeling of the atria further increases the likelihood of thrombus formation. All of the molecules mentioned are regulated by NO upstream. ${ }^{6 / 4)}$ However, NO appears to be present in different expression levels between the LA and the RA. ${ }^{4)}$ Hence, in this study, we measured the expression level of these molecules and examined whether differences in the expression level of NO translate into differences in the expression level of thrombosis-associated molecules to explain the differential thrombogenesis between the LA and the RA. According to several studies, the condition of an abnormality in the tissue factor, one of the three conditions in Virchow's triad, is better fulfilled by the LA than the RA. Goldsmith et al. ${ }^{211}$ observed microscopic alterations of the two chambers and reported that cellular and intercellular perforations in the endocardium were more frequently seen in the LA. Modrego et al. ${ }^{22}$ reported observations at the molecular level, suggesting that the RA is better protected from harmful agents than the LA as evidenced by higher expression of cytoprotective proteins like heat shock protein 27 (Hsp27) and glutathione-S-transferase in the RAA. However, the extensive analysis of factors associated with thrombogenesis in our study proved otherwise. The mRNA or protein levels of factors such as E-selectin, CD31, as well as ET-1 were not significantly different between the LA and the RA. Our findings enable us to exclude abnormality in the vessel wall as a cause for increased thrombi formation in the LA.

\section{Pathophysiological differences between the left atrium and the right atrium in atrial fibrillation-associated thromboembolism}

Even though both $L A$ and $R A$ can be seen fibrillating during $A F$, 
the $L A$, rather than the $R A$, is predominantly responsible for initiation of fibrillatory activity as well as intracardiac thrombogenesis. ${ }^{23)}$ If there is no significant difference in the tissue factors responsible for thrombogenicity, then what is the potential mechanism for the difference in thrombogenicity between the LA and the RA? There are several potential mechanisms for the LA-RA difference in thromboembolic risk. First, the RA appendage (RAA) is pyramidal in shape, whereas the LAA is a finger-like extension. The expression of transcription factors and left-right differential signaling ${ }^{24)}$ lead to a number of morphological differences between the LA and the RA. ${ }^{25)}$ The difference in the shape carries multiple ramifications. The volume of the LAA is significantly smaller, ${ }_{1}^{26)}$ and the LAA ostium is much narrower than the RAA ostium. ${ }^{27)}$ Ultimately, the differences in morphology and junction with their respective atria cause undisturbed blood flow in the RAA and stasis in the LAA. Second, more extensive remodeling of the LA may have resulted in a greater loss of atrial contractility on the left side of the heart, forcing the blood to pool to a greater extent when in the LA. The degree of fibrosis and extracellular matrix remodeling was greater in the LAA than in the RAA. ${ }^{28)}$ At the molecular level, the matrix metalloproteinase (MMP)-9 level was higher in the LAA than in the RAA, which explains the greater degradation of the extracellular matrix and subsequent chamber dilatation and wall thinning in the LA than in the RA. ${ }^{22)}$ Third, due to the nature of connection of each chamber to the surrounding organs, clinical consequences of thrombi from the two atria also differ. Lungs have thrombolytic mechanisms that can dissolve throm$\mathrm{bi}$, and are rich in the collateral vascular network; hence thrombi from the RA may lead to little clinical consequence or subclinical pulmonary embolism. In contrast, however, brain has a sparse anastomotic vascular network and has minimal antithrombotic mechanism, thus making the thrombi originating in the LA more important from a clinical perspective as well as in further studies.

\section{Limitations}

A small sample size and the selection bias might be the limitations of this study. The atrial appendage tissues were taken based on the surgeon's decision during the operation, and only those patients who underwent an atrial biopsy were included in this study. Both the RA and LA tissues were taken from the same 6 out of the 25 enrolled patients. Although we included relatively homogeneous patients with mitral valve disease and the comparative results of these 6 patients were consistent to the overall analyses, it may have affected the results of this study. Although the main purpose of the current study was to perform a comparison between the LA and the RA, we could not compare volumes of the LA and RA based on imaging studies. Because the cardiac CT protocol was designed for left cardiac chamber evaluation and limited contrast agent filling in the RA, an accurate measurement of the RA volume was not possible in this patient population.

In conclusion, in spite of more advanced subendocardial or matrix remodeling in the $L A$ than in the $R A$, the biomarkers representing endocardial function were not significantly different between the RA and the LA in patients with mitral valvular AF.

\section{Acknowledgments}

This research was supported by a grant from the Korean Society of Cardiology (2011) and the Korea Health 21 R\&D Project (A120478), Ministry of Health and Welfare in Republic of Korea.

\section{References}

1. Sohara $H_{1}$ Amitani $S$, Kurose M, Miyahara K. Atrial fibrillation activates platelets and coagulation in a time-dependent manner: a study in patients with paroxysmal atrial fibrillation. J Am Coll Cardiol 1997;29: 106-12.

2. Li-Saw-Hee FL, Blann AD, Lip GY. Effect of degree of blood pressure on the hypercoagulable state in chronic atrial fibrillation. Am J Cardiol 2000;86:795-7, A9.

3. Freestone B, Lip GY. The endothelium and atrial fibrillation. The prothrombotic state revisited. Hamostaseologie 2008;28:207-12.

4. Cai $H$, Li Z, Goette $A$, et al. Downregulation of endocardial nitric oxide synthase expression and nitric oxide production in atrial fibrillation: potential mechanisms for atrial thrombosis and stroke. Circulation 2002;106:2854-8.

5. Dudley SC Jr, Hoch NE, McCann LA, et al. Atrial fibrillation increases production of superoxide by the left atrium and left atrial appendage: role of the NADPH and xanthine oxidases. Circulation 2005;112: 1266-73.

6. Sung ML, Wu CC, Chang HI, et al. Shear stress inhibits homocysteineinduced stromal cell-derived factor-1 expression in endothelial cells. Circ Res 2009;105:755-63.

7. Malmström RE, Settergren M, Pernow J. Endothelin attenuates endothelium-dependent platelet inhibition in man. Acta Physiol (Oxf) 2010; 198:441-8.

8. Abi-Younes S, Sauty A, Mach F, Sukhova GK, Libby P, Luster AD. The stromal cell-derived factor-1 chemokine is a potent platelet agonist highly expressed in atherosclerotic plaques. Circ Res 2000;86:131-8.

9. Chen $C N$, Chang SF, Lee PL, et al. Neutrophils, lymphocytes, and monocytes exhibit diverse behaviors in transendothelial and subendothelial migrations under coculture with smooth muscle cells in disturbed flow. Blood 2006;107:1933-42.

10. Urbich C, Dimmeler S. Endothelial progenitor cells: characterization and role in vascular biology. Circ Res 2004;95:343-53.

11. Frenette PS, Moyna C, Hartwell DW, Lowe JB, Hynes RO, Wagner DD. Platelet-endothelial interactions in inflamed mesenteric venules. Blood 1998;91:1318-24.

12. Rosenblum WI, Murata $S$, Nelson GH, Werner PK, Ranken R, Harmon RC. Anti-CD31 delays platelet adhesion/aggregation at sites of endo- 
thelial injury in mouse cerebral arterioles. Am J Pathol 1994;145:33-6.

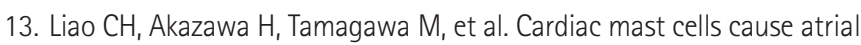
fibrillation through PDGF-A-mediated fibrosis in pressure-overloaded mouse hearts. J Clin Invest 2010;120:242-53.

14. Ohkita $M$, Takaoka $M$, Shiota $Y$, Nojiri $R$, Matsumura $Y$. Nitric oxide inhibits endothelin-1 production through the suppression of nuclear factor kappa B. Clin Sci (Lond) 2002;103 Suppl 48:68S-71S.

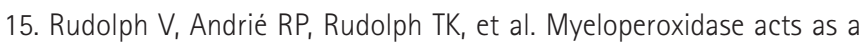
profibrotic mediator of atrial fibrillation. Nat Med 2010;16:470-4.

16. Park JH, Pak HN, Lee S, Park HK, Seo JW, Chang BC. The clinical significance of the atrial subendocardial smooth muscle layer and cardiac myofibroblasts in human atrial tissue with valvular atrial fibrillation. Cardiovasc Pathol 2013;22:58-64.

17. Mortazavi A, Williams BA, McCue $K$, Schaeffer L, Wold B. Mapping and quantifying mammalian transcriptomes by RNA-Seq. Nat Methods 2008;5:621-8.

18. De Caterina R, Libby $P$, Peng $H B$, et al. Nitric oxide decreases cytokineinduced endothelial activation. Nitric oxide selectively reduces endothelial expression of adhesion molecules and proinflammatory cytokines. J Clin Invest 1995;96:60-8.

19. Turu MM, Slevin M, Matou S, et al. C-reactive protein exerts angiogenic effects on vascular endothelial cells and modulates associated signalling pathways and gene expression. BMC Cell Biol 2008;9:47.

20. Burstein $B$, Libby $E$, Calderone $A$, Nattel $S$. Differential behaviors of atrial versus ventricular fibroblasts: a potential role for platelet-derived growth factor in atrial-ventricular remodeling differences. Circulation 2008;117:1630-41.

21. Goldsmith I, Kumar P, Carter P, Blann AD, Patel RL, Lip GY. Atrial endocardial changes in mitral valve disease: a scanning electron microscopy study. Am Heart J 2000;140:777-84.

22. Modrego J, Maroto L, Tamargo J, et al. Comparative expression of proteins in left and right atrial appendages from patients with mitral valve disease at sinus rhythm and atrial fibrillation. J Cardiovasc Electrophysio/ 2010;21:859-68.

23. Fuster $V$, Rydén $L E$, Asinger RW, et al. ACC/AHA/ESC guidelines for the management of patients with atrial fibrillation: executive summary. $A$ Report of the American College of Cardiology/American Heart Association Task Force on Practice Guidelines and the European Society of Cardiology Committee for Practice Guidelines and Policy Conferences (Committee to Develop Guidelines for the Management of Patients With Atrial Fibrillation): developed in Collaboration With the North American Society of Pacing and Electrophysiology. J Am Coll Cardiol 2001;38: 1231-66.

24. Franco D, Campione M. The role of Pitx2 during cardiac development. Linking left-right signaling and congenital heart diseases. Trends Cardiovasc Med 2003;13:157-63.

25. King T, Bland Y, Webb S, Barton S, Brown NA. Expression of Peg1 (Mest) in the developing mouse heart: involvement in trabeculation. Dev Dyn 2002;225:212-5.

26. Corradi $D$, Maestri $R$, Macchi $E$, Callegari $S$. The atria: from morphology to function. J Cardiovasc Electrophysiol 2011;22:223-35.

27. Ho SY, McCarthy KP. Anatomy of the left atrium for interventional electrophysiologists. Pacing Clin Electrophysio/ 2010;33:620-7.

28. Subramaniam B, Riley MF, Panzica PJ, Manning WJ. Transesophageal echocardiographic assessment of right atrial appendage anatomy and function: comparison with the left atrial appendage and implications for local thrombus formation. J Am Soc Echocardiogr 2006;19:429-33. 